\title{
Autophagy contributes to the chemo- resistance of non-small cell lung cancer in hypoxic conditions
}

\author{
Jin Gu Lee ${ }^{1}$, Ju Hye Shin ${ }^{2}$, Hyo Sup Shim³ ${ }^{3}$ Chang Young Lee ${ }^{1}$, Dae Joon Kim¹, Young Sam Kim²+ \\ and Kyung Young Chung ${ }^{1,4^{*}+}$
}

\begin{abstract}
Background: The development of chemo-resistance in non-small lung cancer is a major obstacle in treating patients. Hypoxia is a commonly faced microenvironment in solid tumor and suggested to be related to both autophagy and chemo-resistance.

Methods: In this study, we investigated the role of hypoxia-induced autophagy in acquiring chemo-resistance in both cancer cell (A549) and human cancer tissue

Results: Hypoxic exposure (1 \% O2) of A549 cell stimulated autophagic induction in cancer cells, shown by increase of LC3BI to LC3BIl conversion and decrease of p62/sequestosome1 in Western blot, increased GFP-LC puncta in confocal microscopy, and increased number of double-membrane autophagic vacuoles in electron micrographs. Hypoxic exposure also induced resistance of cancer cells to cisplatin, and LC3B siRNA restored the sensitivity of cancer cells to chemotherapy. Furthermore, Human lung cancer tissues that experienced chemotherapy showed increase of LC3BI to LC3BII conversion and decrease of p62/sequestosome1 compared with chemo-naïve cancer tissue in Western blot.
\end{abstract}

Conclusion: Autophagy may play an important role in acquiring resistance to chemotherapy in lung cancer and hypoxia related pathway seems to be involved in autophagy induction.

Keywords: Autophagy, Chemotherapy, Hypoxia, Lung cancer, Resistance

\section{Introduction}

Lung cancer is a common cause of cancer-related death worldwide [1]. Non-small cell lung cancer (NSCLC) composes more than $80 \%$ of lung cancer and chemotherapy is a major treatment option for NSCLC patients. However, even the best regimens have an overall response rate of only $30-50 \%$. This poor response to chemotherapy leads to the treatment failure in lung cancer.

Autophagy is considered to have dual functions in cancer as both a tumor suppressor and a protector of tumor cell survival $[2,3]$. Before a tumor develops, autophagy suppresses the tumor; however, once the tumor begins to develop, autophagy supports the tumor in

\footnotetext{
* Correspondence: kychu@yuhs.ac

${ }^{\dagger}$ Equal contributors

'Department of Thoracic and Cardiovascular Surgery, Yonsei University College of Medicine, Seoul, South Korea

${ }^{4} 50$ Yonsei-ro, Seodaemun-gu, Seoul 120-752, South Korea

Full list of author information is available at the end of the article
}

harsh microenvironments. Some studies found that autophagy is up-regulated in response to cancer treatments, and protects tumor cells by contributing to the development of treatment resistance. This observation implies that pharmacological modulation of autophagy can be a therapeutic target for tumor cells [2-6].

Tumor cells often face hasher microenvironments than normal cells. Hypoxia occurs in $90 \%$ of solid tumors because angiogenesis in tumor cannot supply rapidly growing tumor masses with enough oxygen [7-10]. Hypoxia also influences several cancer-related pathways including angiogenesis signaling, cell migration, energy metabolism, cell growth, and damage deoxyribonucleic acid (DNA) repair. Recent studies found relationships between hypoxia-related pathways and autophagy activation. These relationships include oxygen-dependent stabilization of hypoxia-inducible factor 1-alpha (HIF- $1 \alpha$ ) transcription factor, inhibition of the mammalian target of 
rapamycin (mTOR) kinase signaling pathway and activation of the unfolded protein response (UPR) [11-13]. HIF- $1 \alpha$ is the main regulator of transcription during hypoxia. HIF-1 $\alpha$ overexpression is frequently observed in tumors and is considered to contribute to tumor cell growth and survival by controlling both glycolysis and angiogenesis. However, the effect of autophagy in tumor cells under hypoxia is not fully understood.

We hypothesized that hypoxia-induced autophagy might contribute to the resistance of NSCLC cells to chemotherapeutic agents by decreasing their apoptotic potential and enhancing their survival. To test this hypothesis, we studied the effect of hypoxia on tumor cells treated with chemotherapeutic agents and analyzed the effects of autophagy. Furthermore, to support our hypothesis, we measured HIF- $1 \alpha$ expression in tumor tissues and autophagy markers in tumor tissues obtained from NSCLC patients.

\section{Materials and methods}

\section{Cell lines and cultures}

The A549 human lung cancer cell line was from the Korean Cell Line Bank just before use and it wasn't validated again. A549 cells were maintained as monolayers in RPMI 1640 medium (GIBCOBRL, Gaithersburg, MD, USA) containing $10 \%$ fetal bovine serum, $100 \mathrm{U} / \mathrm{ml}$ penicillin (Thermo Scientific, Rockford, IL, USA) and $0.1 \mathrm{mg} / \mathrm{ml}$ streptomycin (Thermo Scientific,) at $37.0{ }^{\circ} \mathrm{C}$ under $5 \% \mathrm{CO}_{2}$.

\section{Reagents}

The chemotherapeutic agent cisplatin (Sigma, St. Louis, MO, USA) was dissolved in RPMI 1640 medium to produce a stock solution $(1 \mathrm{mM})$ and added directly to media at indicated concentrations.

\section{Hypoxia treatment}

For induction of hypoxic conditions, cells $(1 \times 104$ cells/ well) were seeded in 96-well flat-bottomed plates overnight, then incubated in hypoxia incubator (Forma Scientific, Ohio, USA) with $1 \% \mathrm{O} 2$.

\section{MTT colorimetric assays}

To investigate the influence of hypoxia on cancer cell chemosensitivity, A549 cells were seeded in 96-well plates at $1 \times 10^{4}$ cells/well and cultured in $1 \%$ hypoxia or normoxia in medium containing cisplatin as indicated. Cell viability was examined with MTT assay kits (Sigma). Spectrophotometric absorbance was measured using a plate reader at $570 \mathrm{~nm}$. All experiments were carried out in triplicate.

\section{Transient transfection and identification of autophagy} A549 cells were seeded $(6.25 \times 105$ cells/well $)$ in 6 -well plates overnight, and a GFP-LC3 expressing plasmid was transiently transfected into cells using Lipofectamine LTX
Reagent (Invitrogen, Carlsbad, CA, USA), according to the manufacturer's instructions. After $24 \mathrm{~h}$ and verifying the expression of GFP-LC3, cells were subjected to hypoxia $\left(1 \% \mathrm{O}_{2}\right)$ for $8 \mathrm{~h}$. After exposure, autophagy (GFP-LC3-positive dots) was counted (GFP-LC3-positive dots) under a confocal laser microscope, LSM700 (Carl Zeiss, Jena, Germany).

\section{Electron microscopy}

Samples were fixed with $2 \%$ glutaraldehydeparaformaldehyde in $0.1 \mathrm{M}$ phosphate buffer (PB), $\mathrm{pH} 7.4$ for $12 \mathrm{~h}$ at $4{ }^{\circ} \mathrm{C}$ and washed three times for $30 \mathrm{~min}$ in $0.1 \mathrm{M}$ PB. Samples were postfixed with $1 \%$ OsO4 dissolved in $0.1 \mathrm{M}$ PB for $2 \mathrm{~h}$ and dehydrated in an ascending gradual series (50-100\%) of ethanol and infiltrated with propylene oxide. Specimens were embedded using a Poly/Bed 812 kit (Polysciences, Warrington, PA, USA). After pure fresh resin embedment and polymerization at $60{ }^{\circ} \mathrm{C}$ in an electron microscope oven (TD-700, DOSAKA, Kyoto, Japan) for $24 \mathrm{~h}$, 350-nm sections were cut and stained with toluidine blue for light microscopy and 70-nm thin sections were double stained with $7 \%$ uranyl acetate and lead citrate for contrast staining. Sections were made with a LEICA Ultracut UCT Ultra-microtome (Leica Microsystems, Wetzlar, Germany). All thin sections were observed by TEM (JEM-1011, JEOL, Tokyo, Japan) at an acceleration voltage of $80 \mathrm{kV}$.

\section{Western blot}

Expression of autophagy-related proteins was analyzed by Western blot. After indicated treatments, A549 cells were lysed in RIPA lysis buffer (Biosesang, Seongnam, Gyeonggi, KOREA) with $1 \mathrm{mM}$ PMSF. Equal amounts of proteins were separated by SDS-PAGE and transferred to PVDF membranes (Millipore Corporation, Billerica, MA, USA). After blocking with $5 \%$ nonfat milk, membranes were probed with anti-HIF-1 $\alpha$ (BD Biosciences, San Jose, California, USA), anti-LC3 (Sigma), anti-p62Cterminus/SQSTM1 (Santa Cruz Biotechnology, Santa Cruz, CA, USA), anti-beclin-1 (Santa Cruz), or anti- $\beta$ actin (Sigma) and developed with ECL (Thermo Scientific, Rockford, IL, USA) reagent.

\section{NSCLC patients and samples}

To determine overexpression of autophagy-related factors after chemotherapy, we selected five NSCLC patients (three adenocarcinoma and two squamous cell carcinoma) who had not yet been treated and five NSCLC patients (two adenocarcinoma and three squamous cell carcinoma) who received neoadjuvant treatment before operation as our study group. All patients underwent complete tumor resection and mediastinal lymph node dissection at the Department of Thoracic and Cardiovascular Surgery of 
Severance Hospital of Yonsei Medical University. The research was approved by the Institutional Review Board of Yonsei University, College of Medicine. All patients gave signed, informed consent. Tumor specimens and surrounding normal lung tissue (separated from tumors by $5 \mathrm{~cm}$ ) were obtained at surgery, or selected by a pathologist, and stored. Specimens were divided into three groups: protein preparation, TEM and IHC.

\section{Immunohistochemisty for Hypoxia expression}

Surgical specimens were immediately transferred from the operating room to the laboratory, fixed in $10 \%$ formaldehyde for four days, and embedded in paraffin. Formalin-fixed and paraffin-embedded tissues were sectioned at 4 um and stained with antibody against HIF- $1 \alpha$ (ab2185, 1:50, Abcam, Cambridge, UK), Carbonic anhydrase IX (CA IX) (ab15086, 1:300, Abcam, Cambridge, UK) and Glucose transporter 1 (GLUT1) (ab652, 1:100, Abcam, Cambridge, UK) using a Ventana automated immunostainer (Ventana Medical Systems, Tucson, AZ) according to the manufacturer's protocol. Signals were detected using a DAB Map detection kit (Ventana Medical Systems) based on the labeled streptavidinbiotin method. The frequency of nuclear staining was evaluated without knowledge of clinical or pathologic status. Five fields (x200) were analyzed to determine the frequency of HIF- $1 \alpha$ stained nuclei. At least 500 tumor cells in the five fields were counted. HIF- $1 \alpha$ staining was evaluated using a semiquantitative grading system based on the number of tumor cells with completely dark stained nuclei within the tumor tissue (0: none, 1 : less than $10 \%$ positivity, $2: 10 \%$ or greater positivity). Occasional cytoplasmic staining was ignored because active HIF- $1 \alpha$ is located only in the nucleus [14]. Stained slides were graded independently by a single pathologist (HS Shim).

\section{Protein extraction and Western blotting of tumor tissues} Tumor tissues and adjacent tissues were homogenized in $1 \mathrm{ml}$ RIPA buffer (50 mM Tris- $\mathrm{HCl}, \mathrm{pH}$ 7.5, $0.1 \%$
SDS, $2 \mathrm{mM}$ EDTA, $150 \mathrm{mM} \mathrm{NaCl}, 1 \%$ sodium deoxycholate, $1 \%$ Triton X-100) and protease inhibitor cocktail. Homogenate was incubated for $20 \mathrm{~min}$ on ice and centrifuged at $14,000 \mathrm{rpm}$ for $15 \mathrm{~min}$ at $4{ }^{\circ} \mathrm{C}$. Supernatant was collected and the same volume of 5X SDS buffer was added. The mixture was boiled for $5 \mathrm{~min}$ and stored at $-80{ }^{\circ} \mathrm{C}$. Proteins were separated by sodium dodecy1 sulfate polyacrylamide gel electrophoresis (SDSPAGE) on $8-16 \%$ polyacrylamide gels and transferred to PVDF membranes (Millipore Corporation, Billerica, MA, USA), Membranes were blocked in $5 \%$ nonfat milk in Tris-buffered saline containing $0.05 \%$ Tween-20 (TBST) at room temperature for $1 \mathrm{~h}$. Membranes were incubated with rabbit monoclonal antibody against LC3 (1:2000 diluted in TBST, Novus, USA) overnight at $4{ }^{\circ} \mathrm{C}$ and washed five times with TBST for $10 \mathrm{~min}$ at room temperature. Membranes were incubated with horseradish peroxidaseconjugated anti-rabbit secondary antibody (1:2000 dilution in TBST, Santa Cruz) at $37{ }^{\circ} \mathrm{C}$ for $1 \mathrm{~h}$ and washed five times with TBST for $10 \mathrm{~min}$ at room temperature. Membranes were treated with ECL (Thermo Scientific, Rockford, IL, USA) reagent and exposed to photographic film. Bands were quantified using densitometry and results were shown as relative expression of the protein from different samples compared to a control sample.

\section{Statistical analysis}

MTT assays were analyzed using SoftMax Pro5.4 (Molecular Devices Corporation, Sunnyvale, CA, USA). All experiments were repeated at least three times. Data were expressed as mean \pm standard error of the mean (SEM). Statistical analysis was performed using Student's $t$-test or ANOVA (two-tailed). The criterion for statistical significance was $p<0.05$.

\section{Results}

Hypoxia induces expression of HIF-1a in cancer cells

A549 cells were exposed to $1 \%$ hypoxia for 2, 4, 6 or $8 \mathrm{~h}$, and HIF- $1 \alpha$ expression was measured to confirm

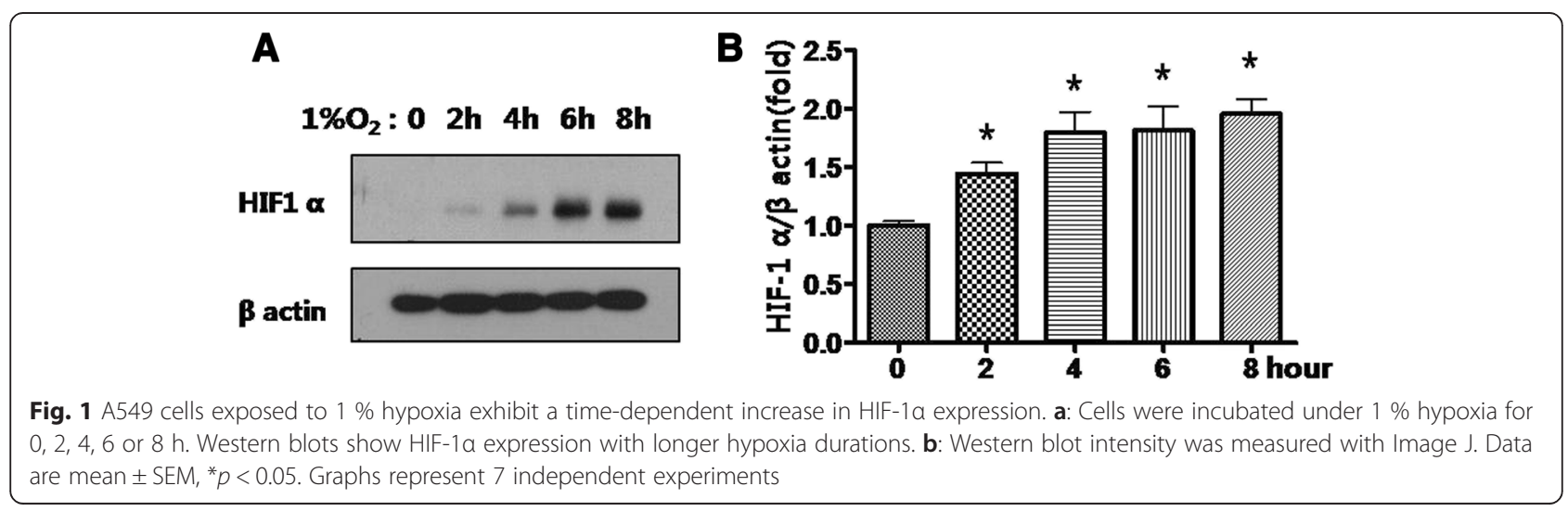




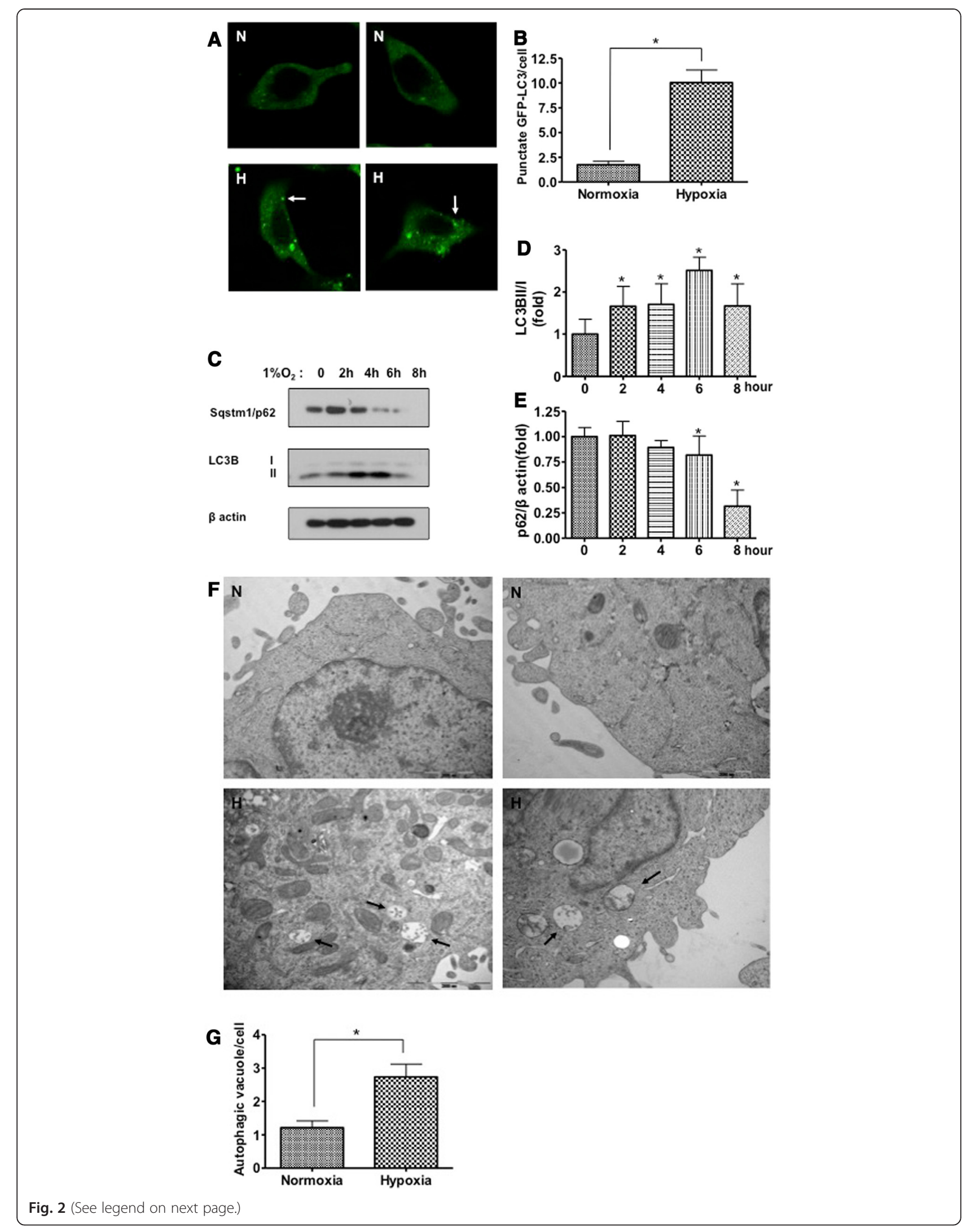


(See figure on previous page.)

Fig. 2 Hypoxia induces autophagy in cancer cells. a: LC3-GFP distribution by confocal microscopy. A549 cells were incubated in normoxia or hypoxia for 8 h. GFP-LC3 showed diffuse intracellular localization under normoxic conditions. Membrane translocation (punctate localization) indicative of autophagy was observed in hypoxic cells (magnification x40). White arrows, punctate GFP-LC3. b: Quantification of GFP-LC3 translocation in transiently transfected normoxic and hypoxic cells. The number of GFP-LC3 spots per cell was counted. c: A549 cells were incubated under normoxia or hypoxia for 2, 4, 6 or $8 \mathrm{~h}$. Western blots showed LC3B-I to LC3B-II conversion in A549 cells incubated in $1 \%$ hypoxia compared with normoxia. Expression of the p62 marker of autophagy flux decreased in A549 cells incubated at $1 \%$ hypoxia compared with normoxia. d, e: Intensity of Western blots measured by Image J. Graphs represents 6 independent experiments. f: Electron micrographs of A549 cell ultrastructure after normoxia or hypoxia for 8 h. Black arrows, double-membraned autophagic vacuoles (magnification $\times 20,000$ ). $\mathbf{g}$ : Quantification of autophagic vacuoles in normoxic and hypoxic cells. The number of autophagic vacuoles per cell was counted. $\mathrm{N}$, normoxia; $\mathrm{H}$, hypoxia. Data are mean $\pm \mathrm{SEM},{ }^{*} p<0.05$

hypoxia at each time point by Western blot. HIF-1 $\alpha$ protein started to increase rapidly within $2 \mathrm{~h}$ of exposure to $1 \%$ hypoxia and increased further with longer exposure (Fig. 1).

\section{Hypoxia induces autophagy in cancer cells}

Next, we measured autophagy activity under hypoxia. First, we measured expression from a vector encoding green fluorescent protein-tagged microtubule-associated protein 1 light chain 3 (GFP-LC3), which condenses in autophagic vacuoles, resulting in punctate fluorescence within cancer cells undergoing autophagy. A549 cells were transiently transfected with GFP-LC3 plasmids. After $24 \mathrm{~h}$, cells were exposed to normoxia or hypoxia. After $8 \mathrm{~h}$ of hypoxia, we counted cells with diffuse or punctate GFP under a confocal microscope. Under normoxia, GFP-LC3 cells were diffusely fluorescent whereas GFP-LC3 cells showed punctate patterns under hypoxia, indicating autophagosome formation (Fig. 2a, b).

Using additional independent assays, we confirmed the involvement of autophagy. We used Western blots to analyze the conversion of LC3B-I to LC3B-II by protein processing, which is a hallmark of autophagy. Levels of endogenous LC3B-I to LC3B-II conversion were substantially increased in A549 cells exposed to $1 \%$ hypoxia compared with cells under normoxia. We also determined levels of p62/sequestosome1 (SQSTM1), the degradation of which is used as an autophagy flux marker. Levels of endogenous p62/SQSTM1 were substantially decreased in A549 cells exposed to $1 \%$ hypoxia compared with cells under normoxia (Fig. 2c, d, e).

Transmission electron microscopy (TEM) was used as another independent assay of autophagy. Cells exposed to hypoxia showed substantial accumulation of autophagosomes with cytoplasmic organelles and other vesicles encapsulated in vacuoles (Fig. 2f, g black arrows), suggesting that hypoxia stimulated autophagy and autophagy was enhanced significantly by hypoxia.

\section{Hypoxia enhances cancer cell chemo-resistance}

To investigate the influence of hypoxia on chemoresistance, A549 cells were exposed to $1 \%$ hypoxia or normoxia for $8 \mathrm{~h}$ in the presence or absence of the chemotherapeutic agent cisplatin. Cell death determined by 3-[4,5-dimethylthiazol-2yl]-diphenyltetrazolium bromide (MTT) assays showed that $32.2 \%$ of cells were dead under normoxia and $11.8 \%$ were dead under $1 \%$ hypoxia with cisplatin $(512 \mu \mathrm{M})$ (Fig. 3). These data suggested that under hypoxic condition, A549 cells were resistant to a chemotherapeutic agent.

\section{Blocking autophagy restores sensitivity of cancer cells to chemotherapy}

To confirm the involvement of autophagy in cancer cells acquiring resistance to chemotherapy under hypoxia, we inhibited autophagy using LC3B siRNA in cisplatintreated A549 cells. The siRNA restored the sensitivity of cancer cell to Cisplatin in hypoxia (Fig. 4a). The blocking of autophagy by siRNA was verified by assaying conversion of reduced LC3-I to II (Fig. 4b). These results suggested that activation of autophagy mediated the protective effect of hypoxia on Cisplatin-induced cell death, and siRNA targeting LC3B restored chemotherapy-induced cell death by suppressing autophagy.

Tumor tissues of lung cancer patients experience hypoxia To confirm the effect of hypoxia on solid tumors, we investigated whether hypoxia was seen in tumors of NSCLC patients. HIF- $1 \alpha$ expression was assessed by both immunohistochemistry (IHC) and Western blot

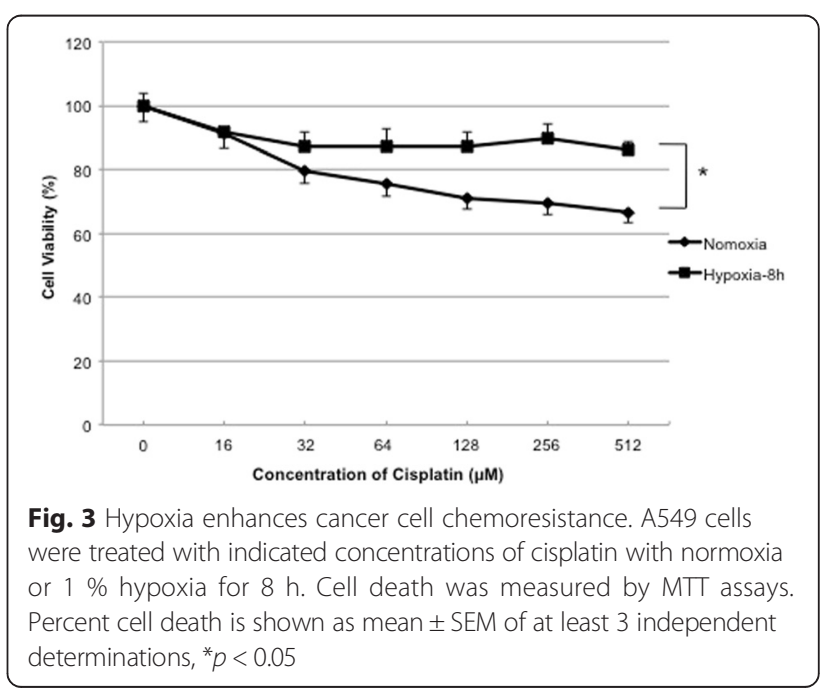



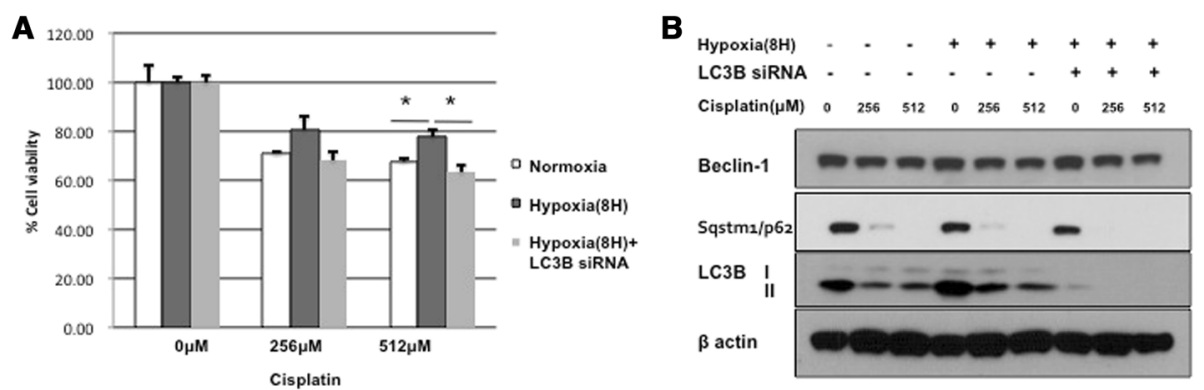

Fig. 4 Knockdown of LC3B restored cancer cells sensitivity to cisplatine under hypoxia. a: A549 cells were transfected with siRNA negative control or siRNA for $L C 3 B$ and treated with indicated concentrations of cisplatin under normoxia or hypoxia for $8 \mathrm{~h}$. Cell death was measured by MTT assays. Percent cell death is shown as mean \pm SEM of at least 3 independent determinations. $\mathbf{b}$ : Western blots of LC3B-I to LC3B-II conversion in A549 cells transfected with LC3B siRNA in hypoxia compared with siRNA negative control under normoxia or hypoxia, ${ }^{*} p<0.05$

and compared with normal lung tissue. Tumors showed higher expression of HIF-1- $\alpha$ compared with normal lung tissue on IHC (1.4 vs. 0.2, $p<0.05)$ (Fig. 5a, b). Western blots also showed higher expression of HIF-1 $\alpha$ in tumors than in normal lung tissue (Fig. 5c, d).

Besides HIF- $1 \alpha$ expression, we also analyzed CA IX and GLUT1 expression by IHC, which are another hypoxia markers, to confirm the hypoxic condition of tumor. All normal tissues showed no expression of both CA IX and GLUT1, but five and eight of ten tumor tissues showed positive expression in CA IX $(p<0.05)$ (Fig. 5e) and GLIT1 $(p<0.05)$ (Fig. 5f) stain respectively. These results indicate that hypoxia is a commonly faced condition in NSCLC tumor.

\section{Chemotherapy enhanced autophagy induction in tumors of lung cancer patients}

To confirm the induction of autophagy in patients with NSCLC after chemotherapy, we analyzed the conversion of LC3B-I to LC3B-II. We also analyzed levels of p62/ SQSTM1 in patients with NSCLC who underwent therapeutic pulmonary resection after neoadjuvant chemotherapy, and patients with NSCLC (chemotherapy naïve) who underwent only therapeutic pulmonary resection. Patients who received chemotherapy before pulmonary resection showed increased LC3B-I to LC3B-II conversion and decreased p62/SQSTM1 compared with patients who were chemotherapy naïve (Fig. 6). These results indicated that autophagy was induced by chemotherapy in the tumor tissue of NSCLC patients.

\section{Discussion}

In this study, we found that hypoxia-induced autophagy resulted in enhanced cancer cell survival after chemotherapy. We also confirmed hypoxia and enhanced autophagy activities in response to chemotherapy in human tumor tissues. To our knowledge, this is the first study that demonstrates the role of autophagy especially in hypoxic condition for the developing chemo-resistance in lung cancer.

In spite of recent advances in chemotherapeutic agents, cancer cells show drug resistance that manifests clinically as tumor progression or recurrence. Although many studies have analyzed drug resistance, the mechanisms associated with chemotherapy resistance remain unknown.

Autophagy is important in cellular homeostasis and facilitating cell survival in adverse conditions [15-19] including hypoxia [20-23]. The effect of autophagy on tumors is controversial. Autophagy functions as both a suppressor and a protector of tumor cells [2, 3]. Specifically, after tumor development, autophagy supports the persistence of the tumor. Autophagy is documented in several cancer cells. It is upregulated in response to therapeutic stress caused by chemotherapy and radiation [4-6]. In our study, we showed that autophagy contributes to chemo-resistance in a lung cancer cell line. We also observed enhanced autophagy activity in post-treatment lung cancer tissue after neo-adjuvant treatment. Most advances in understanding autophagy and cancer are from studies using cancer cell lines. Little information is available about autophagy in clinical tumor samples. To investigate our hypothesis, we measured autophagy activity in treatmentnaïve tumors and post-treatment tumors from NSCLC patients after neo-adjuvant treatment. This report describes enhanced autophagy activity in human lung cancer tissue after treatment.

Among several conditions that induce autophagy, hypoxia is common microenvironments in solid tumors. Hypoxia is often found in solid tumors including lung cancer and is important because it triggers crucial pathways related cancer progression. Cells adapt to hypoxia through several pathways including metastasis, angiogenesis, autophagy and glycolytic metabolism [24]. Recent studies showed that hypoxia induces autophagy [21-23], and autophagy induced by hypoxia protects tumor cells from apoptosis after chemotherapeutic agent 


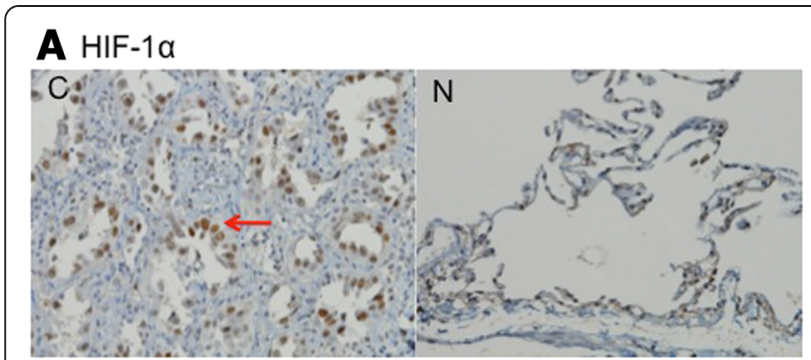

\section{B}

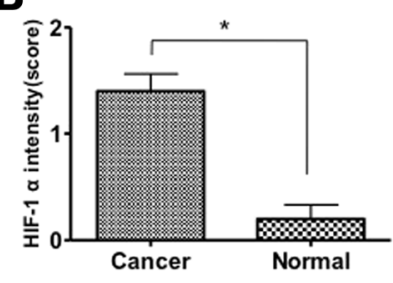

C

Ceoadjuvant tissue

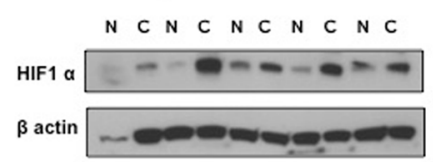

D

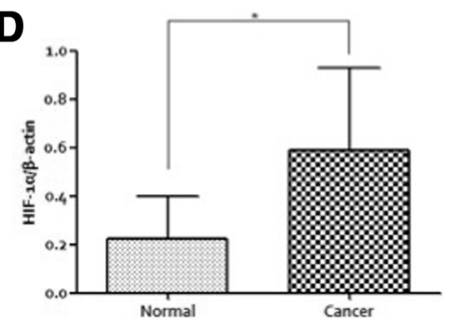

\section{E CAIX}

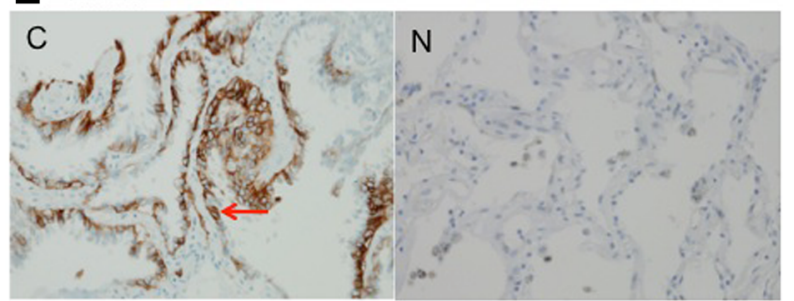

F GLUT1

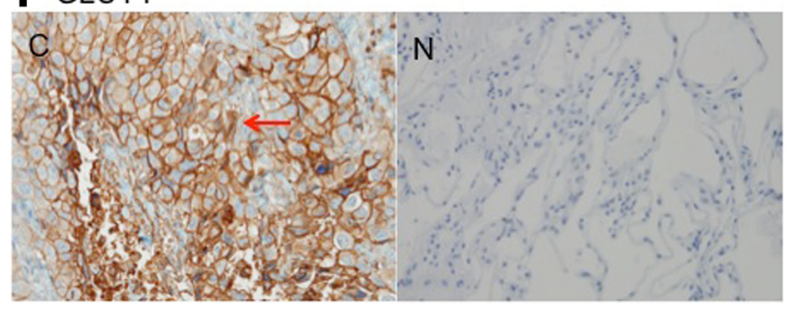

Fig. 5 Hypoxia markers of tumor tissues of non-small cell lung cancer (NSCLC). a: Immunohistochemical (IHC) stain of tumor tissues shows higher hypoxia inducible factor 1 a (HIF-1a) expression than normal lung tissue. Red arrow, HIF-1a expression in tumor cell nucleus (magnification x200). C, cancer; N, normal lung. b: HIF-1a staining scored by a semiquantitative grading system based on tumor cells showing completely dark stained nuclei: 0 , none; 1 , less than $10 \%$ positivity; 2, $10 \%$ or greater positivity. Tissues from ten patients were used for analysis. c: Western blots show higher expression of HIF-1a in tumor tissues of NCSLC. Tumor tissues from patients after treatment or with chemonaïve tumors showing HIF-1a expression compared with normal lung tissue. N, normal; C, cancer. $\mathbf{d}$ : Intensity of Western blots measured using Image J. Tissues from ten patients were used. Data are mean $\pm S E M,{ }^{*} p<0.05$. e. Carbonic anhydrase IX (CA IX) expression by IHC. Five of ten tumor tissues showed positive expression, but no normal tissue showed positive expression ( $p<0.05$ ). Red arrow: CA IX expression (magnification x200). C, cancer; N, normal lung. f. Glucose transporter 1 (GLUT1)) expression by IHC. Eight of ten tumor tissues showed positive expression, but no normal tissue showed positive expression $(p<0.05)$. Red arrow: GLUT1 expression (magnification x200). C, cancer; N, normal lung

treatment. Aiming the underlying mechanism of hypoxia, the HIF-1 $\alpha$-related pathway is crucial [25-29]. HIF-1 $\alpha$, which is induced by hypoxia, is a main transcription factor and induces autophagy by controlling expression of the downstream targets BNIP3 and BNIP3L [30-32]. We found high expression of HIF- $1 \alpha$ in tumor tissues from NSCLC patients, indicating that hypoxia in solid tumors was involved in the tumor microenvironment. In addition, lung cancer cells exposed to hypoxia were resistant to the chemotherapeutic agent.
Autophagy activities in human solid tumor compared to normal tissue have been reported in several tumors. Beclin 1 and LC3 were decreased in human lung cancer but this expression was not associated with gender, smoking, histological type and stage [33] and decreased expression of LC3 was reported in ovarian cancer [34]. However, Yoshioka et al. [35] reported an increased expression of LC3-II in esophageal and gastrointestinal tumors, and Karpathiou et al. [36] reported LC3A as a prognostic factor in lung cancer. In this study, conversion 


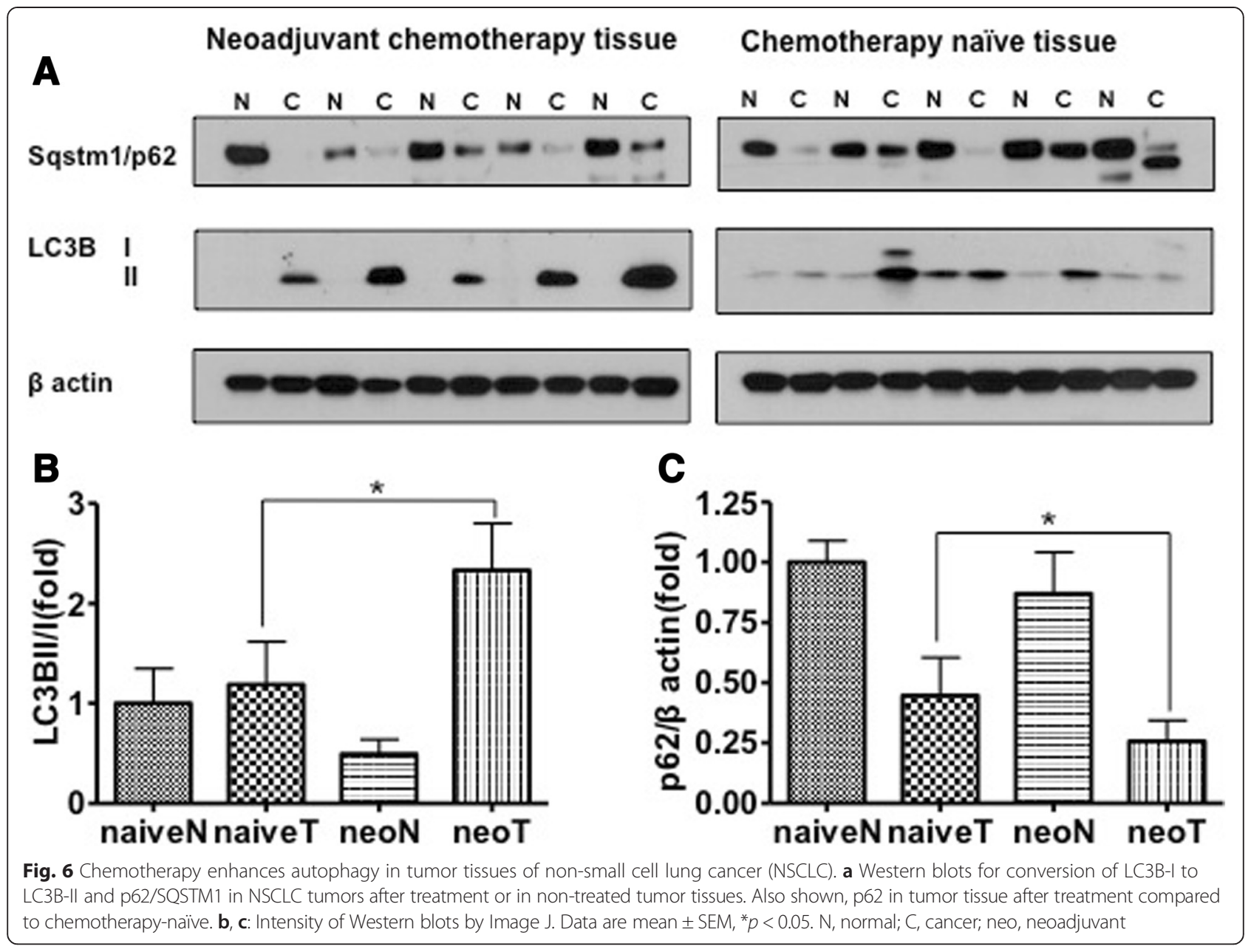

of LC3B-I to LC3B-II couldn't show any difference between naïve tumor tissues and naive normal tissues in Fig. 6. Therefore, the expression of autophagy activity itself in several tumors seems not to be clearly understood yet, and it's surrounding environments such as exposure of chemotherapy or radiotherapy look more important as shown in this study. But further analyses with large number of cases are needed.

This study has some limitations. Only single tumor cell line (A549) and single chemotherapeutic agent (cisplatin) was tested, further experiment with additional cell line and other chemotherapy agents are needed to confirm this finding. On top of that, this study focused on hypoxia induced autophagy activity related to chemoresistance. However, hypoxia is also considered to be associated with inhibition of DNA repair or cell proliferation that could lead to alter cisplatin sensitivity [37]. Further experiments including several hypoxia related pathway are needed to understand the underline mechanism of this hypoxia related chemo-resistance.

Since hypoxia and autophagy are vital for tumor progression and effective cancer treatments, we predict that drugs that alter the hypoxia-autophagy pathway might be beneficial for improving the responsiveness of chemotherapy in cancer patients. Our study demonstrated that autophagy is pivotal in development of drug resistance in NSCLC, especially in hypoxic conditions.

\section{Conclusions}

Autophagy may play an important role in acquiring resistance to chemotherapy in lung cancer and hypoxia related pathway seems to be involved in autophagy induction.

\section{Abbreviations}

ANOVA: Analysis of variance; BCL2: B-cell lymphoma 2; BNIP3: BCL2/adenovirus E1B 19 kDa interacting protein 3; BNIP3L: BCL2/adenovirus E1B 19 kDa interacting protein 3-like; CA IX: Carbonic anhydrase IX; DNA: Deoxyribonucleic acid; GFP: Green fluorescent protein; GFP-LC3: Green fluorescent protein-tagged LC3; GLUT1: Glucose transporter 1; HIF-1a: Hypoxia-inducible factor 1-alpha; IHC: Immunohistochemistry; LC3: Microtubule-associated protein 1 light chain 3; MTT: 3-[4,5-dimethylthiazol-2yl]-diphenyltetrazolium bromide; mTOR: Mammalian target of Rapamycin; NSCLC: Non-small cell lung cancer; siRNA: Small interfering RNA; RNA: Ribonucleic acid; SEM: Standard error of the mean; SQSTM1: Sequestosome1; TEM: Transmission electron microscope; UPR: Unfolded protein response. 


\section{Competing interest}

No potential conflicts of interest were disclosed.

\section{Authors' contributions}

$J$ developed the conception and methodology, performed the statistical analysis and drafted the manuscript. JS carried out the Western blotting studies and cell experiments. HS carried out immunohistochemistry staining and analysis. CL provided surgical specimens and participated in acquisition of data. DK provided surgical specimens. YK helped conceive the study, helped with interpretation of data, coordination it and oversaw the manuscript. KC participated in the design of the study, and coordinated it and helped revise the manuscript. All authors read and approved the final manuscript.

\section{Acknowledgements}

This study was supported by a faculty research grant of Yonsei University College of Medicine for 2011 (6-2011-0148).

\section{Author details \\ ${ }^{1}$ Department of Thoracic and Cardiovascular Surgery, Yonsei University College of Medicine, Seoul, South Korea. ${ }^{2}$ Department of Internal Medicine, Yonsei University College of Medicine, Seoul, South Korea. ${ }^{3}$ Department of Pathology, Yonsei University College of Medicine, Seoul, South Korea. ${ }^{4} 50$ Yonsei-ro, Seodaemun-gu, Seoul 120-752, South Korea.}

Received: 2 April 2015 Accepted: 23 September 2015

Published online: 09 November 2015

\section{References}

1. Jemal A, Siegel R, Ward E, Hao Y, Xu J, Thun MJ. Cancer statistics, 2009. CA Cancer J for clin. 2009;59:225-49.

2. Chen N, Karantza-Wadsworth V. Role and regulation of autophagy in cancer. Biochim Biophys Acta. 1793;2009:1516-23.

3. White E, DiPaola RS. The double-edged sword of autophagy modulation in cancer. Clin Cancer Res. 2009;15:5308-16.

4. Kondo $Y$, Kanzawa $T$, Sawaya $R$, Kondo S. The role of autophagy in cancer development and response to therapy. Nat Rev Cancer. 2005;5:726-34.

5. Mathew R, Karantza-Wadsworth V, White E. Role of autophagy in cancer. Nat Rev Cancer. 2007;7:961-7

6. Lum JJ, Bauer DE, Kong M, Harris MH, Li C, Lindsten T, et al. Growth factor regulation of autophagy and cell survival in the absence of apoptosis. Cell. 2005; 120:237-48.

7. Cosse JP, Michiels C. Tumour hypoxia affects the responsiveness of cancer cells to chemotherapy and promotes cancer progression. Anticancer Agents Med Chem. 2008;8:790-7.

8. Zhou J, Schmid T, Schnitzer S, Brüne B. Tumor hypoxia and cancer progression. Cancer Lett. 2006;237:10-21.

9. Höckel M, Vaupel P. Tumor hypoxia: definitions and current clinical, biologic, and molecular aspects. J Natl Cancer Inst. 2001;93:266-76.

10. Semenza GL. Targeting HIF-1 for cancer therapy. Nat Rev Cancer. 2003:3:721-32.

11. Qie Y, Li P Ji C. Cell Death Conversion under Hypoxic Condition in Tumor Development and Therapy. Int JMil Sci. 2015;16(10):25536-51.

12. Rouschop KM, Wouters BG. Regulation of autophagy through multiple independent hypoxic signaling pathways. Curr Mol Med. 2009;9(4):417-24.

13. Mazure NM, Pouysségur J. Hypoxia-induced autophagy: cell death or cell survival? Curr Opin Cell Biol. 2010;22(2):177-80.

14. Lee CH, Lee MK, Kang CD, Kim YD, Park DY, Kim JY, et al. Differential expression of hypoxia inducible factor-1 alpha and tumor cell proliferation between squamous cell carcinomas and adenocarcinomas among operable non-small cell lung carcinomas. J Korean Med Sci. 2003;18:196-203.

15. Papandreou I, Lim A, Laderoute K, Denko N. Hypoxia signals autophagy in tumor cells via AMPK activity, independent of HIF-1, BNIP3, and BNIP3L. Cell Death Differ. 2008;15:1572-81.

16. Abedin M, Wang D, McDonnell M, Lehmann U, Kelekar A. Autophagy delays apoptotic death in breast cancer cells following DNA damage. Cell Death Differ. 2006;14:500-10.

17. Sato K, Tsuchihara K, Fujii S, Sugiyama M, Goya T, Atomi Y, et al. Autophagy is activated in colorectal cancer cells and contributes to the tolerance to nutrient deprivation. Cancer Res. 2007;67:9677.
18. Degenhardt K, Mathew R, Beaudoin B, Bray K, Anderson D, Chen G, et al. Autophagy promotes tumor cell survival and restricts necrosis, inflammation, and tumorigenesis. Cancer cell. 2006;10:51-64.

19. Katayama M, Kawaguchi T, Berger M, Pieper R. DNA damaging agentinduced autophagy produces a cytoprotective adenosine triphosphate surge in malignant glioma cells. Cell Death Differ. 2006;14:548-58.

20. Zakeri Z, Lockshin RA. Cell death: history and future. Adv Exp Med Biol. 2008;615:1-11.

21. Zhang H, Bosch-Marce M, Shimoda LA, Tan YS, Baek JH, Wesley JB, et al. Mitochondrial autophagy is an HIF-1-dependent adaptive metabolic response to hypoxia. J Biol Chem. 2008;283:10892-903.

22. Pursiheimo J, Rantanen K, Heikkinen P, Johansen T, Jaakkola P. Hypoxia-activated autophagy accelerates degradation of SQSTM1/p62. Oncogene. 2008;28:334-44.

23. Tracy K, Dibling BC, Spike BT, Knabb JR, Schumacker P, Macleod KF. BNIP3 is an RB/E2F target gene required for hypoxia-induced autophagy. Mol Cell Biol. 2007;27:6229-42.

24. Schmaltz C, Hardenbergh PH, Wells A, Fisher DE. Regulation of proliferationsurvival decisions during tumor cell hypoxia. Mol Cell Biol. 1998;18:2845-54.

25. Semenza GL, Wang GL. A nuclear factor induced by hypoxia via de novo protein synthesis binds to the human erythropoietin gene enhancer at a site required for transcriptional activation. Mol Cell Biol. 1992;12:5447-54.

26. Hiraga T, Kizaka-Kondoh S, Hirota K, Hiraoka M, Yoneda T. Hypoxia and hypoxia-inducible factor-1 expression enhance osteolytic bone metastases of breast cancer. Cancer Res. 2007;67:4157.

27. Higgins DF, Kimura K, Iwano M, Haase VH. Hypoxia-inducible factor signaling in the development of tissue fibrosis. Cell Cycle. 2008;7:1128.

28. Smith TG, Robbins PA, Ratcliffe PJ. The human side of hypoxia-inducible factor. Br J Haematol. 2008;141:325-34.

29. Schnitzer S, Schmid T, Zhou J, Brüne B. Hypoxia and HIF-1a protect A549 cells from drug-induced apoptosis. Cell Death Differ. 2006;13:1611-3.

30. Pore N, Jiang Z, Gupta A, Cerniglia G, Kao GD, Maity A. EGFR tyrosine kinase inhibitors decrease VEGF expression by both hypoxia-inducible factor (HIF)-1-independent and HIF-1-dependent mechanisms. Cancer Res. 2006;66:3197-204.

31. Maiuri MC, Criollo A, Tasdemir E, Vicencio JM, Tajeddine N, Hickman JA, et al. BH3-Only proteins and $\mathrm{BH} 3$ mimetics induce autophagy by competitively disrupting the interaction between Beclin 1 and $\mathrm{BCl}-2 / \mathrm{BCl}-\mathrm{X}(\mathrm{L})$. Autophagy. 2007:3:374-6.

32. Oberstein A, Jeffrey PD, Shi Y. Crystal structure of the BCI-XL-Beclin 1 peptide complex. J Biol Chem. 2007;282:13123-32.

33. Jiang ZF, Shao L, Wang WM, Yan XB, Liu RY. Decreased expression of Beclin-1 and LC3 in human lung cancer. Mol Biol Rep. 2012;39(1):259-67.

34. Shen Y, Li DD, Wang LL, Deng R, Zhu XF. Decreased expression of autophagy-related proteins in malignant epithelial ovarian cancer. Autophagy. 2008;4(8):1067-8.

35. Yoshioka A, Miyata H, Doki Y, Yamasaki M, Sohma I, Gotoh K, et al. LC3, an autophagosome marker, is highly expressed in gastrointestinal cancers. Int J Oncol. 2008;33(3):461-8.

36. Karpathiou G, Sivridis E, Koukourakis MI, Mikroulis D, Bouros D, Froudarakis $\mathrm{ME}$, et al. Light-chain 3A autophagic activity and prognostic significance in non-small cell lung carcinomas. Chest. 2011;140(1):127-34.

37. Scanlon SE, Glazer PM. Multifaceted control of DNA repair pathways by the hypoxic tumor microenvironment. DNA Repair (Amst). 2015;32:180-9.

\section{Submit your next manuscript to BioMed Central and take full advantage of:}

- Convenient online submission

- Thorough peer review

- No space constraints or color figure charges

- Immediate publication on acceptance

- Inclusion in PubMed, CAS, Scopus and Google Scholar

- Research which is freely available for redistribution 